\title{
An islanding detection strategy for distribution network connected with hybrid DG resources
}

\author{
J.A. Laghari ${ }^{\text {a,d }}$, H. Mokhlis ${ }^{\text {a,b,*, }}$, M. Karimi ${ }^{\text {a }}$, A.H.A. Bakar ${ }^{\text {b }}$, Hasmaini Mohamad ${ }^{c}$ \\ a Department of Electrical Engineering, Faculty of Engineering, University of Malaya, 50603 Kuala Lumpur, Malaysia \\ ' University of Malaya Power Energy Dedicated Advanced Centre (UMPEDAC), Level 4, Wisma R\&D UM, University of Malaya, Jalan Pantai Baharu, \\ 59990 Kuala Lumpur, Malaysia \\ 59990 Kuala Lumpur, Malaysia
c Faculty of Electrical Engineering, University of Technology MARA (UiTM), 40450 Shah Alam, Selangor, Malaysia \\ 'Faculty of Electrical Engineering, University of Technology MARA (UiTM), 40450 Shah Alam, Selangor, Malaysia
d Department of Electrical Engineering, Quaid-e-Awam University of Engineering Science \& Technology, Nawabshah, 67480 Sindh, Pakistan
}

\section{A R T I C L E I N F O}

Article history:

Received 4 November 2013

Received in revised form

18 November 2014

Accepted 9 February 2015

Available online 27 February 2015

Keywords:

Islanding detection techniques

Hybrid Distributed Generation

Average rate of change of reactive power

Bio-Mass

Mini-hydro

\begin{abstract}
A B S T R A C T
The exponential growth in electricity demand has driven the Distributed Generation (DG) technology a boost in the power system. The use of DG is beneficial to power utilities, DG owners', and end-users in terms of reliability, power quality, and economics. However, to fully utilize the benefits of DGs, some technical issues need to be addressed. Islanding condition is one of the most important issue in this context. Until now, several islanding detection techniques have been proposed for detecting the islanding condition. This paper presents a brief overview of existing islanding detection techniques with their relative merits and demerits. Apart from this, the paper presents an islanding detection strategy suitable for hybrid DG resources of mini-hydro and Bio-Mass. The proposed strategy uses average rate of change of reactive power and load shift strategy to detect islanding of the distribution network. The performance of proposed strategy is validated on various islanding and non-islanding events. The results of proposed strategy are compared with other existing techniques in terms of fast islanding detection, and non-detection zone. The simulation results show that the proposed strategy is effective in detecting islanding phenomenon possesses fast detection and negligible non-detection zone region compared to existing islanding detection techniques.
\end{abstract}

Contents

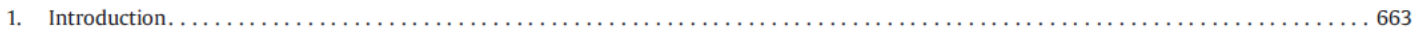

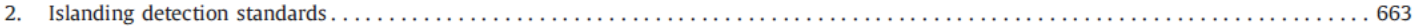

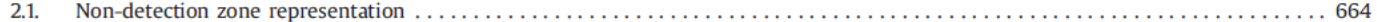

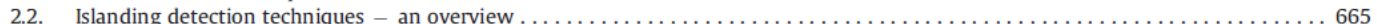

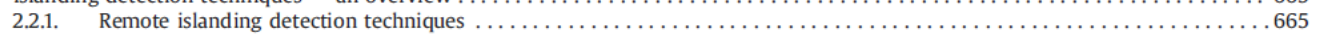

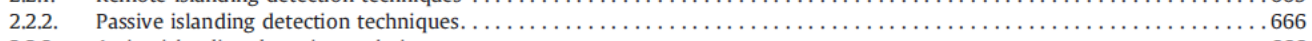

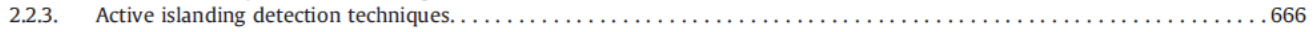

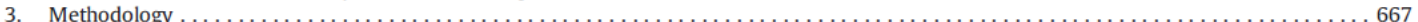

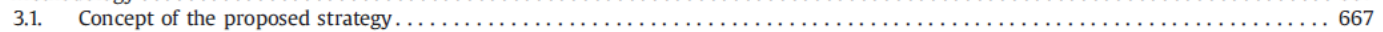

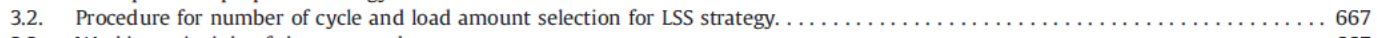

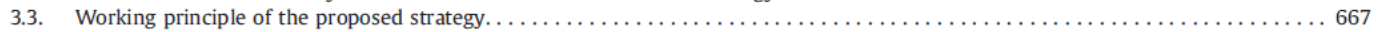

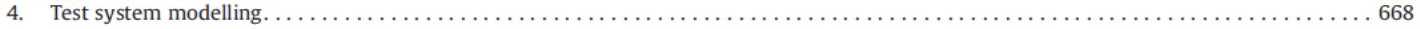

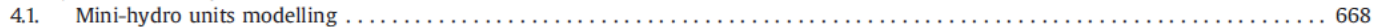

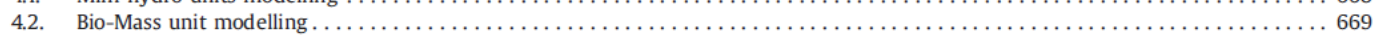

\footnotetext{
* Corresponding author.

E-mail addresses: javedahmedleghari@gmail.com (J.A. Laghari).
} hazli@um.edu.my (H. Mokhlis).

http://dx.doi.org/10.1016/j.rser.2015.02.037

1364-0321/0 2015 Elsevier Ltd. All rights reserved. 


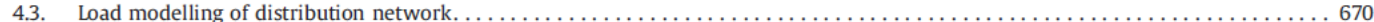

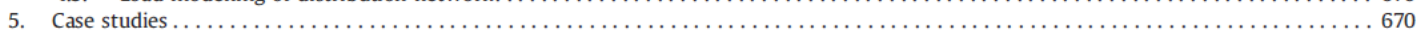

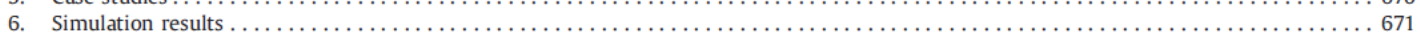

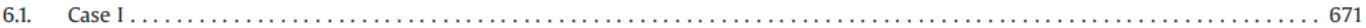

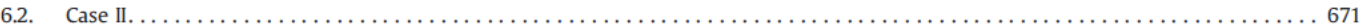

6.3. Case III . . . .

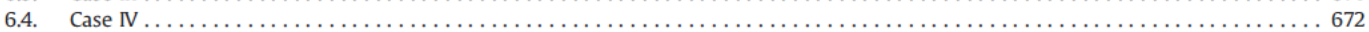

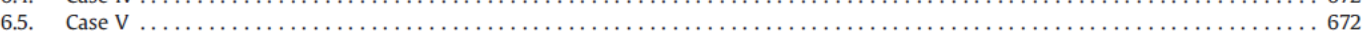

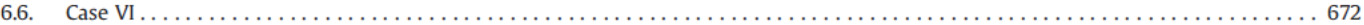

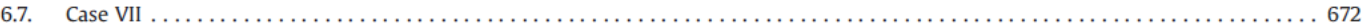

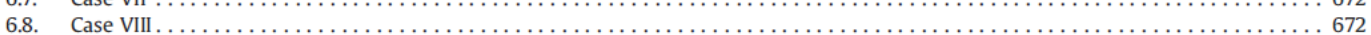

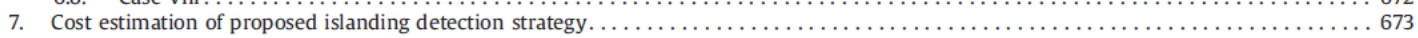

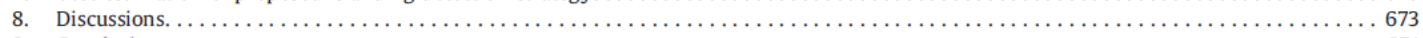

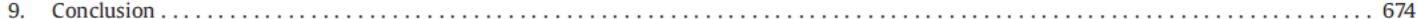

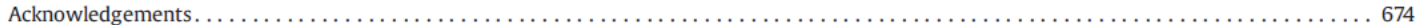

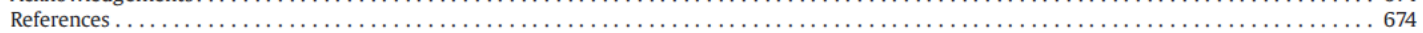

\section{Introduction}

From the last decade, renewable energy resources have been intensively utilized as Distributed Generation (DG) due to global concern on environmental protection and deregulation in electrical power industry $[1,2]$. At present, the DG has been widely employed as the alternative option for electric power generation both from power quality and system reliability point of view [3,4]. Thus, the DG concept represents a paradigm shift from centralized power generation to Distributed Generation [5,6]. A DG technology commonly consists of small-scale power generating resources like small gas turbine, micro-turbine, Bio-Mass, wind, photovoltaic, and fuel cell. The DG owner has the advantage to produce electric power with or without grid connected mode and can sell the surplus power to power utility during off-peak hours $[3,7,8]$.

Many power utilities around the world already possess a significant DG penetration in their networks. Hence, the power utilities can supply that power to customers which may help to avoid the frequency reduction and interruption duration within the distribution network [9]. However, there are still various issues to be addressed before DG can be utilized fully as an integral part of power utilities. The most important issue with the DG is islanding condition. Islanding is a situation in which distribution network loss the grid connection yet continue to be supplied by the DG connected to it. This condition can occur due to various reasons such as tripping of transmission line, equipment failure, operator mistake, and maintenance purposes. This is undesirable due to following reasons [10].

(1) The islanding can create safety hazard to the person working for maintenance. Thus, it may threat line workers safety.

(2) The islanding operation of distribution network may cause severe fluctuations of voltage and frequency of the distribution network, which may result in failure and damage of electrical equipments.

(3) The islanding condition may result in asynchronous reclosing problem between the DG and grid reconnection. This may result in damaging the equipment or re-tripping the line.
(4) With grid disconnection, the power quality of the electric service may be degraded resulting in malfunctioning of the sensitive equipments.

Due to the above reasons, IEEE standard 1547 [11] has stated that in case of such situation, the DG should detect the loss of grid connection and disconnect itself from the distribution network within $2 \mathrm{~s}$ (100 cycles) of the islanding state occurrence. Thus, an efficient and reliable islanding detection technique is required to solve this problem. The researchers have proposed various techniques for islanding detection. This paper presents a brief overview of traditional islanding detection methods with their advantages and disadvantages and proposed a new islanding detection technique to address this issue.

The paper is organized as follows. Section 2 discusses the islanding detection standards, non-detection zone representation, and reviews different existing islanding detection techniques with their merits and demerits. Sections 3 and 4 discusses the methodology of the proposed technique and modelling of the test system whereas; case studies and simulation results are discussed in Section 5 and 6 . Section 7 and 8 presents the cost estimation and discussions of the proposed technique, and in Section 9, conclusion is presented.

\section{Islanding detection standards}

The islanding phenomenon may cause potential damage to electrical equipments, malfunctioning of the sensitive equipments and may threat line workers safety. To prevent these issues, several islanding detection standards have been developed to provide guidelines to researchers for designing the islanding detection methods. These standards include IEEE Std. 1547-2003 [11], IEEE Std. 929-2000 [12], UL 1741 [13], IEC 62116 [14], and VDE 0126-1-1. Table 1 summarizes the standard parameters limit of quality factor, islanding detection time, frequency, and voltage for these standards

Table 1

Standards for islanding detection.

\begin{tabular}{lllll}
\hline Standard & Quality factor, $Q_{f}$ & Islanding detection time, $t$ & Frequency range & Voltage range \\
\hline IEC 62116 & 1 & $t<2 \mathrm{~s}$ & $\left(f_{o}-1.5 \mathrm{~Hz}\right) \leq f$ and $f \leq\left(f_{o}+1.5 \mathrm{~Hz}\right)$ & $85 \% \leq V \leq 115 \%$ \\
UL 1741 & 2.5 & $t<2 \mathrm{~s}$ & $59.3 \mathrm{~Hz} \leq f \leq 60.5 \mathrm{~Hz}$ & $88 \% \leq V \leq 110 \%$ \\
IEEE 1547 & 1 & $t<2 \mathrm{~s}$ & $59.3 \mathrm{~Hz} \leq f \leq 60.5 \mathrm{~Hz}$ & $88 \% \leq V \leq 110 \%$ \\
Korean standard & 1 & $t<0.5 \mathrm{~s}$ & $59.3 \mathrm{~Hz} \leq f \leq 60.5 \mathrm{~Hz}$ & $88 \% \leq V \leq 110 \%$ \\
IEEE 929-2000 & 2.5 & $t<2 \mathrm{~s}$ & $59.3 \mathrm{~Hz} \leq f \leq 60.5 \mathrm{~Hz}$ & $88 \% \leq V \leq 110 \%$ \\
VDE 0126-1-1 & 2 & $t<0.2 \mathrm{~s}$ & $47.5 \mathrm{~Hz} \leq f \leq 50.5 \mathrm{~Hz}$ & $88 \% \leq V \leq 110 \%$ \\
\hline
\end{tabular}




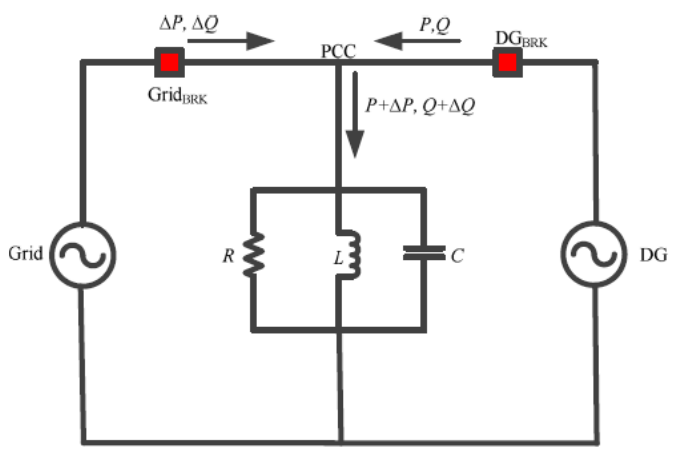

Fig. 1. Standard test frame configuration for islanding detection.

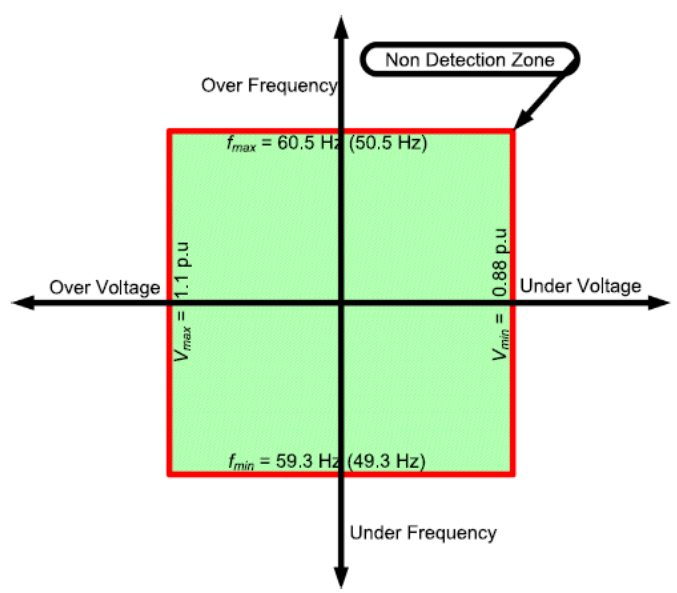

Fig. 2. Non-detection zone by OUV/OUF method.

$[15,16]$. To validate the islanding detection technique for the above prescribed limits, a standard test circuit configuration shown in Fig. 1 is commonly used. In this configuration, islanding event can be simulated by opening the grid breaker. The performance of the islanding detection method can be tested for various islanding and non-islanding events to satisfy the typical limits of voltage, frequency, and time to detect islanding.

\subsection{Non-detection zone representation}

The non-detection zone is considered as an important factor for determining the accuracy and effectiveness of islanding detection technique. It is the range in terms of power difference between generation and load demand where an islanding detection technique fails to detect the islanding. The islanding detection technique with smallest non-detection zone is more accurate than with large non-detection zone. The non-detection zone can be represented in terms of frequency and voltage range as well as active power and reactive power mismatch. The NDZ for active power is given by the following equation $[17,18]$ :

$\Delta P=-3 V \times \Delta V \times I$

where $\Delta P$ is the active power imbalance, $V$ is the rated voltage, $I$ is the rated current, and $\Delta V$ is the voltage deviation (difference of $V_{\max }$ and $V_{\min }$ ). Th, 18]:

$\Delta Q=3 \frac{V^{2}}{\omega_{n} L}\left(1-\frac{f_{n}^{2}}{\left(f_{n} \pm \Delta f\right)^{2}}\right)$

where $\Delta Q$ is the reactive power imbalance, $V$ is the rated voltage, $f_{n}$ is the nominal frequency, and $\Delta f$ is the frequency deviation (difference of $f_{\max }$ and $f_{\min }$ ).

$\omega_{n}=2 \times \pi \times f$,

$L=V^{2} / 2 \times \pi \times f \times Q_{f} \times P$

where $Q_{f}$ is the quality factor, which is the ratio of the amount of energy stored in the load's reactive elements to the amount of energy dissipated in the load's resistance. It is given by

$Q_{f}=R \sqrt{C / L}$

The value of quality factor varies from 2.5 to 1 for different islanding detection standards as shown in Table 1 . A $Q_{f}$ value of 2.5 represents a power factor of 0.37 which is the worst case of low power factor in practical systems. The quality factor is inversely proportional to power factor of the system. The non-detection zone for standards over/under frequency and over/under voltage based islanding detection technique according to Table 1 is shown in Fig. 2.

It can be noticed from Fig. 2 that if islanding occur within this mentioned range of voltage and frequency, then it will not be detected by the conventional methods. Another way to calculate the non-detection zone in terms of active and reactive power mismatch is obtained by using the following equations [19]:

$\left(V / V_{\max }\right)^{2}-1 \leq \Delta P / P \leq\left(V / V_{\min }\right)^{2}-1$

$Q_{f} \times\left(1-\left(f / f_{\min }\right)^{2}\right) \leq \Delta Q / Q \leq Q_{f} \times\left(1-\left(f / f_{\max }\right)^{2}\right)$

where $V_{\max }$ is the maximum limit of over voltage, $V_{\min }$ is the minimum limit of under voltage, $f_{\max }$ is the maximum limit of over

Table 2

Typical value for standard parameters.

\begin{tabular}{llll}
\hline Parameter & Value & Parameter & Value \\
\hline$V_{\max }$ & $1.1 \mathrm{p.u}$ & $Q_{f}$ & 2.5 \\
$V_{\min }$ & $0.88 \mathrm{p.u}$ & $V$ & $1 \mathrm{p.u}$ \\
$f_{\max }$ & $60.5 \mathrm{~Hz}$ & $f$ & $60 \mathrm{~Hz}$ \\
$f_{\min }$ & $59.3 \mathrm{~Hz}$ & - & - \\
\hline
\end{tabular}

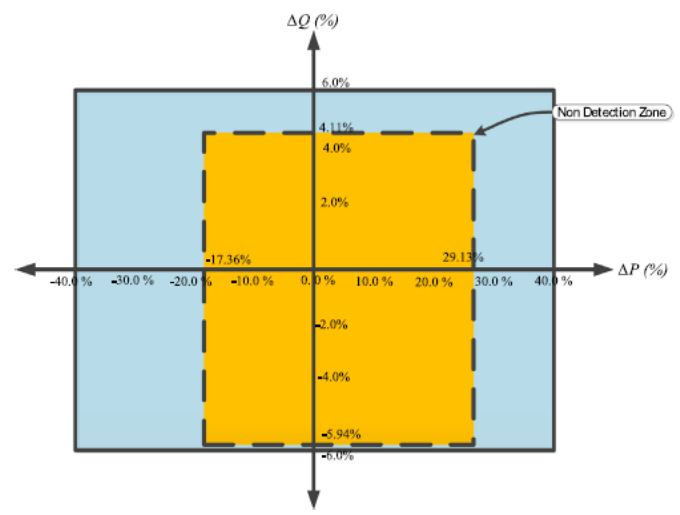

Fig. 3. NDZ in terms of active and reactive power mismatch (\%). 
frequency, $f_{\min }$ is the minimum limit of under frequency, $Q_{f}$ is the quality factor, $V$ is the rated voltage, $f$ is the rated frequency, $P$ is the active power, $Q$ is the reactive power, $\Delta P$ is the active power mismatch, and $\Delta Q$ is the reactive power mismatch.

By putting typical values of standard parameters as given in Table 2 in the above equations, it becomes

$-17.36 \% \leq \frac{\Delta P}{P} \leq 29.13 \%$

$-5.94 \% \leq \frac{\Delta Q}{Q} \leq 4.11 \%$

Eqs. (5) and (6) shows the typical values of NDZ in terms of active and reactive power mismatch for over/under voltage and over/under frequency based methods. Fig. 3 shows the NDZ region of active and reactive power mismatch based on above values. It can be noticed from Fig. 3 that reactive power mismatch is more sensitive (6\% with $\left.Q_{f}=2.5\right)$ than active power mismatch $\left(29 \%\right.$ with $\left.Q_{f}=2.5\right)$ [19]. Salles et al. [20] have proposed another practical method to accurately estimate the non-detection zones of conventional frequency and voltage based islanding detection techniques for synchronous based DG. The technique has the advantage that NDZ can be estimated with a few analytical expressions without relying on numerous, time consuming simulations.

\subsection{Islanding detection techniques - an overview}

Up to now, various islanding detection technique have been proposed, which can be broadly classified into remote and local islanding detection techniques. Local islanding detection techniques are further sub-classified into passive and active islanding detection methods [21-26]. A comprehensive overview of these islanding detection methods has been presented in $[27,28]$. However, up to now, most of the islanding detection techniques have been proposed and discussed from the academic point of view. Hence, many of them despite of interesting features are complicated to implement and might not be practical for real applications. In this perspective, Guo et al. [29] have presented a comprehensive overview of those islanding detection methods that has been used in the US patents for grid-connected inverters [29]. The classification of islanding detection techniques with their types is shown in Fig. 4. The details of each islanding detection techniques are discussed in the following sections.

\subsubsection{Remote islanding detection techniques}

The principle of remote islanding detection techniques is based on the information transfer through communication means between the DG and electric utility. In these techniques, when islanding occurs, a communication signal is sent to trip the DG units. The basic operating principle of remote islanding detection techniques is shown in Fig. 5.

It can be observed from Fig. 5 that the transmitter (installed at utility) continuously sends the signal to receiver (installed at DG) through power line carrier. However, in case of islanding occurrence, this communication medium is broken and receiver does not get any signal. Hence, by this way, islanding is detected. This remote technique is known as power line signalling scheme because it uses the power line carrier as communication medium between the utility and DG [30-32]. Transfer trip scheme is also another type of remote technique which uses supervisory control

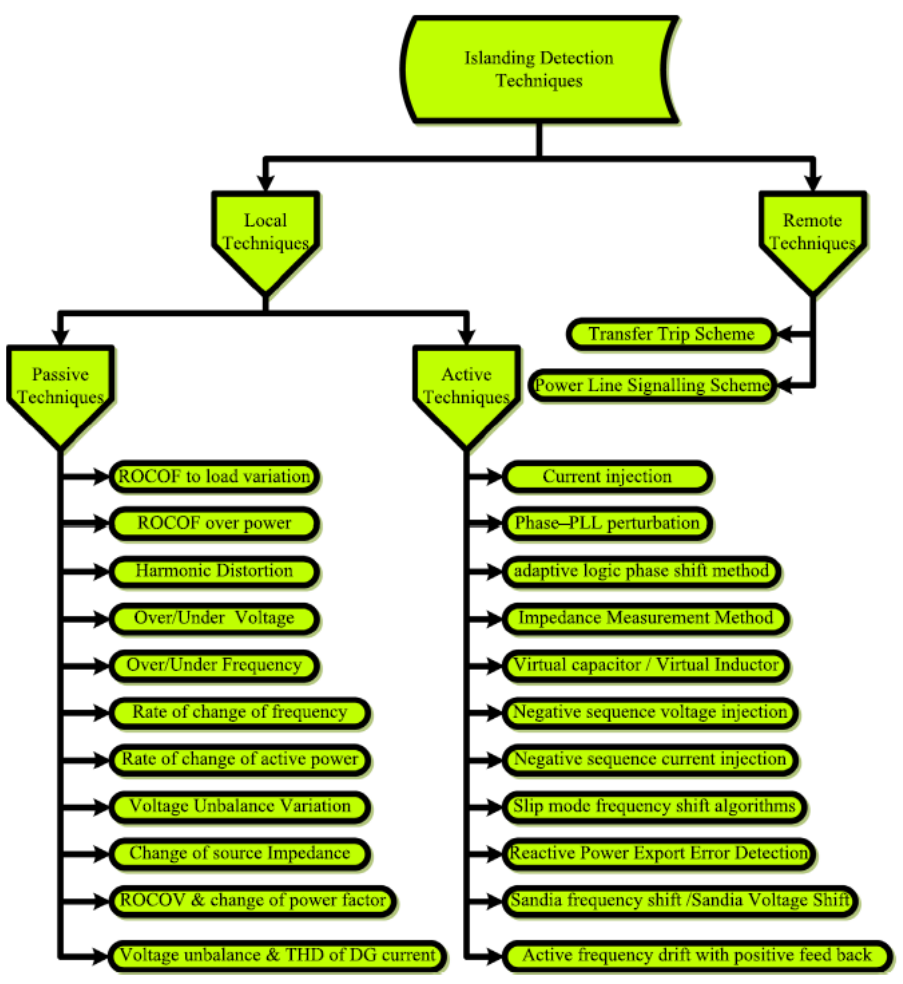

Fig. 4. Classification of islanding detection techniques. 
and data acquisition (SCADA) system as a communication medium for islanding detection [33].

Remote islanding detection techniques have better reliability than local techniques and are quite useful for multiple DG operation. However, these techniques are very much expensive compared to local islanding detection methods. Their implementation is not economical for small distribution networks [25,34]. Due to these reasons, the research has shift towards local islanding detection techniques which are cost effective option for islanding detection compared to remote technique.

2.2.2. Passive islanding detection techniques

The principle of passive islanding detection techniques is based on measurement of system parameters and comparing it with threshold value. If the measured value exceeds the threshold value, islanding is detected. The examples of passive technique are shown in Fig. 4. The passive techniques have the following advantages:

(1) These techniques do not affect the power quality of the distribution network.

(2) These techniques are cost effective and easy to implement and have short islanding detection time.

However, passive techniques have major drawback of possessing very large non-detection zone (NDZ). Furthermore, these techniques need special care in setting the threshold values because low threshold setting may cause nuisance tripping whereas, high threshold setting may cause failure in islanding detection. However, efforts have been done to reduce the NDZ of the passive islanding detection techniques by combining it with artificial intelligence techniques. Alam et al. [35] have combined the support vector machine (SVM) with conventional vector surge (VS) and rate of change of frequency relay to reduce their NDZ. The technique obtained features of voltage, frequency, and rotor angle by generating numerous sets of offline dynamic simulations under different operating conditions. The extracted features are then used as inputs to a support vector machine to classify the event as islanding or non-islanding. The simulation results indicated that the technique possessed accuracy of $98 \%$ and above [35]. Similarly, application of support vector machine for enhancement in the performance of passive islanding detection technqiue has been proposed in [36-38]. Apart from these, probabilistic neural network [39] and artificial neural network [40] are also utilized in the passive islanding detection techniques to enhance their performance.

Some researchers have used different approaches to improve the performance of passive islanding detection techniques. Abbey et al. [41] have presented an auto-ground approach for islanding detection for all type of DGs. For this, a prototype system was constructed using standard distribution apparatus and a recloser controller. The technique was tested on the utility's distribution test line. The results show that when the auto-ground was applied, the DG was disconnected within 1 cycle on over current protection. The technique is promising due to its relatively low cost [41]. Other efforts to enhance the performance of passive islanding detection technique are based on capacitor insertion method [42], inter-locking method based on impedance estimation [43], and empirical mode decomposition method [44]. The results have shown that with the aid of these methods, the performance of passive islanding detection techniques is enhanced and their NDZ can be reduced to almost zero. However, most common way of solving the drawbacks of passive islanding techniques is obtained by using active islanding detection techniques [21-24,45-47].

\subsubsection{Active islanding detection techniques}

Active techniques are based on the injection of high frequency signals or some other means to slightly perturb the system variables for detecting the islanding phenomenon. The main philosophy of

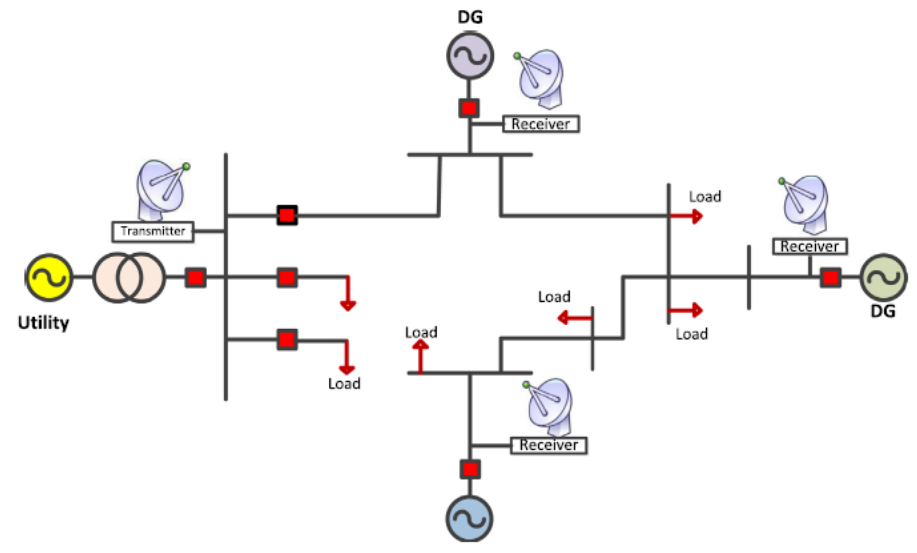

Fig. 5. Operating principle of remote islanding detection technique.

Table 3

Comparison of remote, passive and active islanding detection techniques.

\begin{tabular}{lllll}
\hline Technique & Principle & NDZ region & Detection time & Power quality \\
\hline Remote technique & Communication between DG and utility system & None & Short & No degradation \\
Passive technique & Monitoring changes in system parameters & Large & Short & Hodegh \\
Active technique & Injecting frequency signals or applying disturbances & Small & Long & Low \\
\hline
\end{tabular}


active techniques lies in the concept that in grid connected mode, the injection of perturbation will cause a small variation in system parameter. However, in the islanded mode, the perturbation injection will cause a significant variation in system parameter which can lead to detection of islanding phenomenon. Fig. 4 shows some examples of active islanding detection techniques.

Active islanding detection techniques possess negligible nondetection zone; however, these techniques inject perturbation at the regular intervals of time in the system. These perturbations degrade the quality of power. Bifaretti et al. [48] have performed the comparison of different islanding detection techniques such as rate of change of frequency method, slip mode frequency Shift (SMS) method, and the combination of both SMS and ROCOF methods. The simulation and experimental results proved that an active technique consisting of both SMS and ROCOF provides the fastest detection time interval compared to individual techniques [48].

Furthermore, the suitability of active techniques is mostly limited to type of DG employed in the system. Hence, an active technique suitable for inverter based DG may not work well for synchronous generator based DG. Another drawback of active techniques is that these techniques work satisfactorily for single DG unit only, they may not work satisfactorily for those distribution systems having multiple DG units [49]. However, efforts have been made to satisfy the operation of active techniques for multiple inverters. In this perspective, Reigosa et al. [50] have presented an active method using high frequency signal injection for multiple inverters. The technique used one inverter as the master that continuously injects a high frequency signal for islanding detection, while the rest inverters use a high frequency current cancellation strategy to detect islanding. With this strategy, interference between converters is avoided, as no high frequency current circulates to/from the other inverters. Simulation and experimental results guarantee the successful detection of islanding for multiple inverters [50]. Another active islanding detection technique for the successful detection of islanding for multiple DG is proposed in [51]. The technique is based on individual estimation of transient stiffness by perturbing the each DG at distinct frequencies. By using this separate perturbation, the technique can be applied to multiple DGs without requiring any communication among them. The simulation results show that the technique is capable of detecting the islanding in multiple DGs [51]. Other active techniques for islanding detection in multiple DGs are proposed in [52-54]. Table 3 summarizes the comparison of all these islanding detection techniques.

Some researchers have combined the features of passive and active technique to obtain the higher reliability [55-59]. In these techniques, the active techniques are used only when passive technique is not able to distinguish the islanding event. In this regard, this paper proposes an islanding detection strategy based on average rate of change of reactive power and load shift strategy (LSS). The methodology of the proposed strategy is discussed in the following section.

\section{Methodology}

\subsection{Concept of the proposed strategy}

As discussed in Section 2.1, the reactive power mismatch is more sensitive than active power mismatch [60]. Hence, the proposed islanding detection strategy uses average rate of change of reactive power $(d Q / d t)$ as a mean to detect islanding. $d Q / d t$ is measured at the bus where DG is connected. The proposed strategy operates in two stages. In the first stage, average magnitude of $(d Q / d t)$ is measured for 5 cycles duration and compared with threshold value to detect islanding for large power mismatches between the DGs and load demand. However, for smaller power mismatch, where $d Q / d t$ value may not be sufficient to distinguish between islanding and other load disturbances; LSS strategy is initiated by closing the contactor for 15 cycles duration to produce the transient in the system. After 15 cycles the LSS strategy is deactivated. This load will be connected at DG side to produce this transient. Due to this transient, rate of change of reactive power will be changed which can be utilized to distinguish between islanding and other load disturbances. The main philosophy in this mechanism is that in grid connected mode, the load addition will cause a small variation in $d Q / d t$; however, in islanded mode, distribution network will experience large $d Q / d t$ which will result in islanding detection. In LSS, the load is switched by employing solenoid operated contactor (SOC) which is used to connect or disconnect the load. These types of contactors are cost effective and offer a very long life.

\section{Procedure for number of cycle and load amount selection for LSS strategy}

The proposed technique measures average rate of change of reactive power for 5 cycles duration in the first step and 15 cycles duration in the second step. This measurement can be easily obtained with very fast speed through micro-controllers. Selecting the number of cycle duration is dependent on the duration of transient, produced by connecting the load to distinguish between the islanding and other load disturbance. Hence, number of cycle or transient time should be set as minimum as possible in order to not affect the power quality of the system. Various simulations have been done to select the suitable number of cycles to produce the transient. After many trial and error simulations, it was found that 15 cycles duration is suitable to produce transient because less than 15 cycles duration will not produce a transient in the system. Due to which, it will not be possible to observe the effect of the transient on the system.

Another important factor considered in this technique is the selection of suitable amount of load, used to produce the transient in the system. The selection of suitable amount of load is very important factor because larger value of load will increase unnecessarily the cost of the technique, whereas very small value of load may be not significant in distinguishing between islanding and other events. Thus, the load amount for LSS should be set as minimum as possible but sufficient to distinguish between the grid connected mode and islanded mode. In order to obtain suitable load amount value, a look up table may be formed by using several load magnitudes and testing them for various islanding and non-islanding events (load increment, load decrement, induction motor starting effect, and DG tripping event). From that look up table, suitable load amount can be selected on the basis of having minimum value but ability to distinguish between islanding and non-islanding events.

\subsection{Working principle of the proposed strategy}

Whenever, the distribution network encounter a disturbance, the average magnitude of $d Q / d t$ is measured for the $N$ cycles duration given by

$A_{q 5}=\left|\frac{1}{N} \sum_{i=1}^{N}\left(\frac{d Q}{d t}\right)_{i}\right|$

The value of $N$ for this study is taken as 5 cycles $(0.1 \mathrm{~s})$. After measuring the $A_{q 5}$, it is compared with $(d Q / d t)_{\min }$ which is threshold value set to initiate the proposed islanding strategy. The

\section{Link to Full-Text Articles :}

\section{http://www.sciencedirect.com/science/article/pii/S1364032115001252}

